\title{
La alfabetización informacional en las bibliotecas universitarias de Brasil: visualización de los niveles de incorporación desde la información publicada en sus sitios web
}

Alejandro Uribe Tirado

\begin{abstract}
Profesor-Investigador Escuela Interamericana de Bibliotecología. Universidad de Antioquia (Medellín-Colombia).Doctorando en Documentación Científica $y$ Bibliotecología.Universidad de Granada (GranadaEspaña) - AUIP
\end{abstract}

La formación en competencias informacionales (Alfabetización Informacional) es uno de los retos más actuales de las bibliotecas universitarias ante la posibilidad de acceso a enormes recursos de información que posibilitan los medios digitales y la necesidad de conocer $y$ aplicar mejores criterios de selección $y$ evaluación de la información para recuperar la más pertinente y de mayor calidad. Frente a esta situación, las bibliotecas universitarias iberoamericanas han ido poco a poco incorporando esta formación bien sea desde programas-cursos directos ofrecidos por la biblioteca o mediante el trabajo colaborativo con docentes $y$ facultades en los currículos de distintas universidades 0 en disciplinas específicas. Este trabajo desde la metodología de análisis de contenidos Web, hace una revisión de la información que presentan las bibliotecas universitarias brasileras sobre esta actividad fundamental de formación, para a partir de esa información, visualizar los niveles de incorporación de las competencias informacionales (Alfabetización Informacional) que desde sus sitios web estarían presentando.

Palabras clave: Alfabetización Informacional; Competencias Informacionales; Bibliotecas Universitarias; Iberoamérica; Brasil; Análisis de Contenidos; Sitios Web.

\section{Information literacy in university libraries of Brazil: viewing levels of incorporation}




\title{
from the information published on their web sites
}

\begin{abstract}
Training in information competencies (Information Literacy) is one of the current challenges of university libraries at the possibilities of access to vast information resources that facilitate digital media, but in turn, given the need to understand and apply best practices in selection and evaluation of information to retrieve the most relevant and highest quality. In this situation, IberoAmerican university libraries (Latin-America, Spain and Portugal) have been slowly incorporating this training either from direct training programs, offered from the library or through collaborative work with teachers and schools in curricula of various universities as a whole or in specific disciplines. This work from the methodology of Web content analysis a review of the information presented by the Brazil university libraries on this core activity of training, for from that information, view the levels of incorporation of information competencies (Information Literacy) that from their web sites would be presenting.
\end{abstract}

Keywords: Information literacy; Information competencies; University libraries; Ibero-America; Brazil, Content analysis, Web sites.

Recebido em03.06.2011 Aceito em 25.01.2012

\section{Introducción}

La formación en competencias informacionales -COMPINFO- o Alfabetización Informacional -ALFIN- es una temática que desde mediados de los años ochenta, con sus debidas precisiones y adaptaciones sobre su alcance y sus aspectos terminológicos, conceptuales y teóricos ha venido desarrollándose en Iberoamérica desde el contexto de las bibliotecas, especialmente desde las bibliotecas universitarias ${ }^{1}$, que es donde en términos generales ha habido más avances, a lo cual diferentes

$1 \quad$ Morales (1985); Gómez Hernández (2000); Gómez Hernández y Pasadas Ureña (2003); Angulo Marcial (2003); Miranda (2004); Vives I Gràcia (2004); Lau et al. (2007); Marzal y Calzada Prado (2007); Pinto y Sales (2007); Area Moreira, Gros y Marzal (2008); Licea de Arenas (2009); Da Silva et al. (2009; 2010); Uribe Tirado $(2010 ; 2011)$, entre otros. Ver bibliografía completa en: <http://alfiniberoamerica.blogspot.comhttp://alfiniberoamerica.blogspot.com>. Consultado: 16/12/2012. 
Web

autores brasileros de manera específica han hecho importantes aportes ${ }^{2}$ como lo han evidenciado, entre otros, los trabajos recopilatorios y de análisis bibliométrico de Lecardelli y Prado (2006), Rocha et al. (2008), Dulziak (2010).

Tras estos más de 25 años de precisiones y adaptaciones que han llevado al paso de la instrucción bibliográfica y la educación de usuarios tradicional hasta llegar hoy al paradigma de la formación en /ALFIN, y sus interrelaciones con otras áreas y prácticas como: la gestión del conocimiento (Melo y Araújo, 2007), y la participación ciudadana y la democracia (Santos, Duarte y Prata, 2008); han dado diferentes momentos de desarrollo, que como se ha indicado en trabajos anteriores (Uribe-Tirado, 2010a-2010b), en el contexto iberoamericano podrían identificarse 5 períodos y uno por venir:

a)pre-Inicio (1985-1994);

b)inicio (1995-1999);

c)pre-Avance (2000-2003);

d)avance (2004-2007); y

e)pre-Posicionamiento (2008 - ...), además de la visualización de un $6^{\circ}$ período denominado: Posicionamiento (a partir 2012 ó $2013 . .{ }^{3}$ ).

Figura 1 - Descripción y autores representativos de los 5 períodos de desarrollo-histórico de COMPINFO/ALFIN Iberoamérica

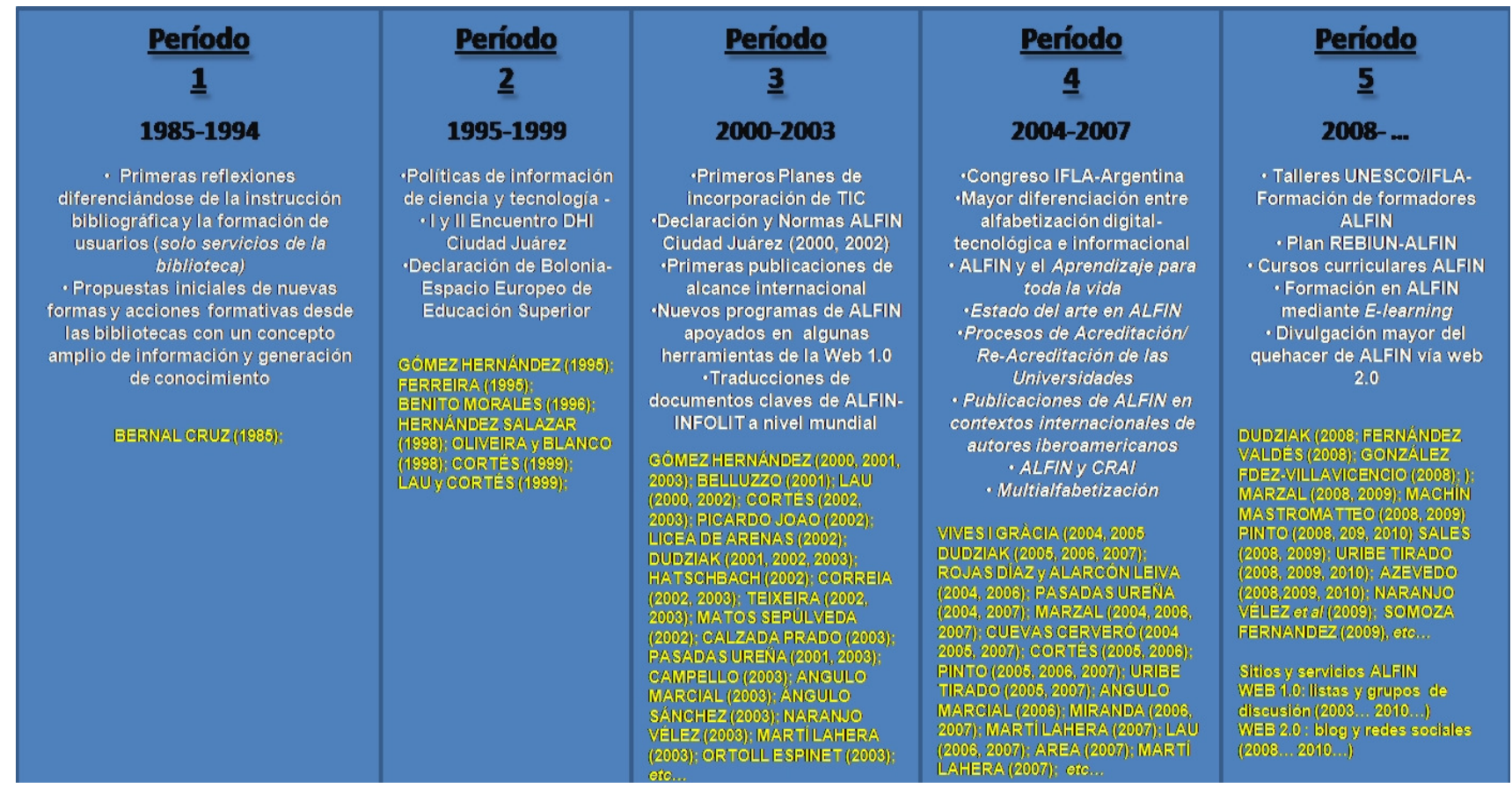

2 Ferreira (1995); Belluzzo (1999; 2003; 2004); Campello (2002; 2003); Caregnato (2000); Dudziak (2001; 2002, 2003; 2008; 2010); Hatschbach (2002; 2008), Dudziak y Ferreira (2004), entre otros. Ver bibliografía completa en: <http://alfiniberoamerica.blogspot.com>. Consultado: 16/12/2012.

3 Este inicio de un $6^{\circ}$ período estará determinado por los buenos resultados de los procesos de incorporación curricular que la ALFIN está viviendo en diferentes Universidades-IES iberoamericanas. 
Fuente: URIBE-TIRADO (2010a; 2010b).

Ante estos procesos de desarrollo, un aspecto clave es identificar cómo se están llevando a cabo al interior de las universidades iberoamericanas la incorporación de esta formación en COMPINFO/ALFIN, para con ello, poder identificar lecciones aprendidas y buenas prácticas que permitan a otras universidades de nuestro contexto iberoamericano, con sus debidas adaptaciones, poder mejorar y avanzar en sus programas de COMPINFO/ALFIN.

Para dicha identificación, una fuente de información clave son los sitios web de las bibliotecas universitarias, ya que en estos momentos, es este medio sin dudas el que más impacto está teniendo en la divulgación de los servicios de las bibliotecas como lo afirman Ferreira Gomes, Prudêncio y Da Conceiçã (2010, p. 147):

A inserção mais ativa da biblioteca na web potencializa as condições de interação com os usuários, redimensionando o seu papel de mediadora do uso e da apropriação da informação. O processo de comunicação consiste no substrato essencial para a mediação da informação [...].

Impacto tanto para los públicos internos que quieren aprovechar su oferta formativa (estudiantes, profesores, investigadores y/o directivos de su universidad a quienes van dirigidos sus programas-cursos de formación en COMPINFO/ALFIN) como para los públicos externos (bibliotecólogos, bibliotecarios, profesores, directivos de otras universidades) que quieren conocer la oferta formativa de esa universidad para realizar procesos de benchmarking, de trabajo cooperativo interinstitucional, de consorcios formativos, etc.

Este trabajo a continuación, en el marco de la investigación: "Lecciones aprendidas en programas de alfabetización informacional en universidades de Iberoamérica" presenta un análisis del caso particular de Brasil $^{4}$, tras el análisis de los sitios web de las bibliotecas universitarias brasileras, la información que presentan, y los niveles en que según esa información se encontrarían en la integración-incorporación de la formación en COMPINFO/ALFIN.

\section{Niveles de integración-incorporación de / ALFIN en las universidades}

Retomando y adaptando la clasificación hecha desde el The landscape concerning the current and future level of ICT integration and elearning in European universities (2004) que reconoce cuatro categorías

\footnotetext{
4Este trabajo se viene realizando país por país en el contexto iberoamericano. Se han publicado ya sus resultados finales o parciales, tanto en revistas científicas como en recursos académicos Web 2.0, para el caso de Colombia y Cuba, y se está en proceso de publicación y edición para los casos de España, Portugal, Puerto Rico, Costa Rica, Perú, Chile, México y Argentina.
} 
de universidades según la incorporación de las TIC:

The frontrunners, The co-operating universities, The self-sufficient universities, The sceptical universities actualizada luego por Finquelevich y Prince (2006), que las clasifican en tres categorías, integrando en una de ellas en dos de las indicadas en el modelo europeo: remisas, emergentes $y$ adelantadas; y sintetizando y ampliando la que proponen Webber y Johnston (2006) específicamente para ALFIN: Embryonic, Intermediate y Threshold ILU -Towards the information literate university-; desde nuestra perspectiva, una universidad o dependencia al interior de la misma (Sistema de Bibliotecas) que deba liderar procesos-programas de formación en COMPINFO/ALFIN puede categorizarse según el grado de incorporación, haciendo una generalización al tener como base las anteriores propuestas, de la siguiente manera (Uribe-Tirado, 2010c):

Universidades-Dependencias comprometidas en la formación en COMPINFO/ALFIN:

Son universidades-dependencias que se caracterizan por un trabajo en COMPINFO/ALFIN de más de una década, en las que Alfabetización Informacional, las competencias informacionales, son definidas tanto en objetivos como en metas concretas en sus Planes Estratégicos, como consecuencia de la concientización sobre su importancia para el aprendizaje para toda la vida y la generación de conocimientos, apoyados en las TIC, el e-learning y en estratégicas didácticas activas, ante las exigencias educacionales-informacionales de la actual sociedad. Por ende, son universidades-dependencias que reconocen la necesidad que las distintas poblaciones de su comunidad universitaria (estudiantes, profesores, investigadores, empleados, directivas) estén adquiriendo esas competencias y tengan diferentes opciones de formación. Opciones de formación de carácter curricular para el caso de los estudiantes, y de educación continua y como requisito de vinculaciónactualización-ascenso en los escalafones para el caso de todos profesores, investigadores, empleados y directivos. Los programas-cursos que conforman estas opciones de formación en COMPINFO/ALFIN, presentan a su vez, unos objetivos y metas de enseñanza-aprendizaje definidos, la adopción de un modelo-norma-estándar de COMPINFO/ALFIN, además de una evaluación permanente de sus resultados (tanto cuantitativos como cualitativos) y un mejoramiento continuo según esos resultados. Las áreas a cargo de los programas o cursos que hacen operativa esta formación, trabajan en forma colaborativa-integrada, y reciben todo el apoyo institucional en recursos financieros-tecnológicos y en capital humano, lo cual 
les permite optimizar esfuerzos y recursos, por lo que los programas-cursos presentan las mejores y más actualizadas posibilidades de acceso, conocimiento y uso a herramientas y servicios especializados de Internet, y de fuentes de información de calidad.

Universidades-Dependencias en crecimiento en la formación en COMPINFO/ALFIN

Son universidades-dependencias que llevan entre 3 y 10 años trabajando en COMPINFO/ALFIN, por lo cual en sus Planes Estratégicos la Alfabetización Informacional y los objetivos y metas que implica, considerando sus diferentes componentes, se están apenas formalizando para ser considerados como claves para su desarrollo institucional. Se comienza a tener conciencia sobre la transversalidad de la COMPINFO/ALFIN en la formación y capacitación de las distintas poblaciones que conforman su comunidad universitaria, pero aún hay muchas instancias al interior de dicha universidad-dependencia que no tienen claras sus implicaciones cognitivas, didácticas, informacionales, tecnológicas, comunicativas. Los cursosprogramas de COMPINFO/ALFIN que ya llevan a cabo, están en proceso de estructuración, definiendo sus objetivos y metas de aprendizaje, y acercándose a la concreción y aplicación de un modelo-norma-estándar de COMPINFO/ALFIN, aunque con pocas experiencias mediante e-learning y didácticas activas; a su vez, comienzan a ser aceptados como parte de los planes curriculares solo en algunas Facultades, y como necesidades de vinculación para los nuevos profesores, investigadores, empleados y directivos. Las evaluaciones de los programascursos que se llevan a cabo en forma más permanente, se centran en lo cuantitativo, aunque ya con algunos avances cualitativos, y no todas las veces generan mejoramientos las valoraciones hechas por los participantes. Se cuentan cada vez con mayores recursos financieros-tecnológicos y de capital humano, pero son insuficientes para las necesidades $y$ requerimientos que las poblaciones universitarias les exigen a las áreas encargadas. Las posibilidades de acceso, conocimiento y uso a herramientas y servicios especializados de Internet, y a fuentes de información de calidad, cada vez aumentan pero aún se quedan cortos para lograr mejores resultados.

Universidades-Dependencias iniciando en la formación en COMPINFO/ALFIN 
Son universidades-dependencias que han comenzado, desde hace 1 a 2 años, a reconocer que los tradicionales programas de formación de usuarios (entrenamiento para el uso de los servicios de la biblioteca) no son suficientes y que la gestión de información y del conocimiento en la actualidad implican el aprendizaje para toda la vida y mayores alcances en esa formación utilizando las grandes posibilidades de los medios digitales y nuevas formas de enseñanza-aprendizaje (elearning y didácticas activas). Por tanto, la COMPINFO/ALFIN no aparece en forma muy definida, formal, entre sus objetivos y metas estratégicas, y se conciben más como cursos aislados o complementarios, no curriculares o de exigencia en adquisición de competencias. Los nuevos cursos de COMPINFO/ALFIN se van construyendo sobre la base de los cursos-programas de formación de usuarios tradicionales, pero poco a poco se comienzan a adicionar o reestructurar pensando en las implicaciones de COMPINFO/ALFIN tanto filosófica como operativamente, y la evaluación se hace presentes esporádicamente para ser parte de presentación de distintos informes desde una perspectiva cuantitativa. Los recursos con que se cuentan son pocos y se está más en la tarea de generar experiencias pilotos, para por medio de sus resultados positivos, ir gestionando nuevos recursos tanto financieros-tecnológicos como de capital humano y de fuentes de información de calidad.

Universidades-Dependencias desconocedoras en la formación en COMPINFO/ALFIN

Son universidades-dependencias donde el tema de COMPINFO/ALFIN, la Alfabetización informacional como concepto no aparece o se confunde con la Alfabetización Digital y se considera que con ésta última es suficiente, que con el acceso a los medios digitales los procesos de aprendizaje permanente y generación de conocimientos se dan por sí solos. Por tanto, en sus Planes Estratégicos si se menciona algún aspecto es enfocado a las tecnologías, a Internet como fin más que como medio. Igualmente se cuenta con programas tradicionales de formación de usuarios, la mayoría de las veces muy precarios, lo cual responde a una concepción muy "corta" de lo que implica una biblioteca o las fuentes de información de calidad para un mejor proceso de enseñanza-aprendizaje, por tanto sus recursos financierostecnológicos, de capital humano y de fuentes de información de calidad son casi nulos. Dichos programas no tienen metas y 
objetivos muy definidos y son más producto de la inercia de varios años estar realizando lo mismo, desde la concepción tradicional de formación de usuarios. Son universidadesdependencias de una tradición más acorde con las universidades del siglo XX que con las exigencias de la sociedad de la información actual.

Estos grados de incorporación de la de formación en COMPINFO/ALFIN pueden sintetizarse e identificarse cuando se analizan los sitios web de las bibliotecas universitarias en diferentes elementos, que podrían resumirse en (Uribe-Tirado, 2011):

Se detecta en el sitio web una sección o información concreta sobre la formación en COMPINFO/ALFIN

Esa sección o información concreta sobre formación en COMPINFO/ALFIN, presenta: (7 macrovariables a valorar)

definición del programa: Misión, Visión, Objetivos, Plan operativo, Plan pedagógico;

fundamentación del programa: Definición de COMPINFO/ALFIN, Modelo pedagógico-didáctico de COMPINFO/ALFIN y Estándar-Indicadores de COMPINFO/ALFIN asumidos;

estructuración del programa: Cursos ofrecidos, Modalidad de los Cursos, Incorporación de las TIC, Web 2.0 y de Ambientes Virtuales de Aprendizaje, Presencia curricular o extracurricular de los Cursos;

evaluación del Programa: Indicadores de gestión, Indicadores de resultados de aprendizaje, Procesos de Mejoramiento continuo.

Además de otra información complementaria, como:

publicaciones sobre este tema y la experiencia de la biblioteca y del programa de COMPINFO/ALFIN: artículos, ponencias, recursos Web 2.0;

participación en grupos y redes de trabajo sobre esta temática: redes de bibliotecas universitarias, colectivos de bibliotecólogos/bibliotecarios, etc.;

participación en medios y recursos de divulgación y aprendizaje de esta temática: listas de discusión, blogs, wikis, twitters, boletines, comunidades virtuales, etc.

Teniendo en cuenta ese marco teórico-conceptual y aplicado que propone 4 niveles incorporación: Comprometidas, en Crecimiento, 
Iniciando y Desconocedoras; y los elementos de contenidos de los sitios Web que permiten el análisis de cómo cada universidad presenta esa incorporación, es posible identificar una correlación que conduce a 4 categorías de incorporación de la formación en COMPINFO/ALFIN según la información Web, que tiene en cuenta a su vez, el proceso de desarrollo en esta temática los últimos años que va de la Instrucción bibliográfica, pasando por la educación de usuarios tradicional hasta llegar a lo que se denomina hoy COMPINFO/ALFIN con sus diferentes niveles.

Esas 4 categorías se concentran principalmente en la variable de Estructuración del programa ya que es la información más fácilmente identificable y más divulgada desde los sitios web de las bibliotecas universitarias (Uribe-Tirado, 2011):

\section{COMPROMETIDAS:}

Alfabetización Informacional. Nivel 2: cursos desde la biblioteca para formar en competencias informacionales: lo instrumental + aprendizaje para toda la vida + pensamiento crítico; y cursos/módulos específicos inmersos oficialmente en los currículos de distintos programas académicos-carreras para formar de manera transversal y disciplinar en esas competencias;

\section{EN CRECIMIENTO:}

Alfabetización Informacional. Nivel 1: cursos desde la biblioteca para formar en competencias informacionales: lo instrumental + aprendizaje para toda la vida + pensamiento crítico;

\section{INICIANDO:}

Formación de Usuarios. Nivel 1: capacitación en servicios generales de la biblioteca y algunos cursos -muy instrumentales- para búsqueda de información: utilización de catálogos/bases de datos, aunque se comienza a analizar la necesidad de cambio de esta formación tradicional y a trabajar las demás competencias;

\section{DESCONOCEDORAS:}

Formación de Usuarios. Nivel 2: solo capacitación para el uso del catálogo 
No hay presencia de ningún tipo de formación-capacitación (treinamento).

Es importante indicar al tener en cuenta este marco metodológico y conceptual-teórico para visualizar los posibles niveles de incorporación de COMPINFO/ALFIN en las bibliotecas universitarias iberoamericanas, que esta categorización es una aproximación desde la información que conllevan los sitios Web, pero en ningún momento implica que categorizar una u otra universidad en alguna de esas 4 categorías sea la total realidad de esa universidad, ya que: "no siempre en los sitios web se publicainforma todo lo que se hace" o por el contrario "se publica-informa más de lo que realmente se hace".

Por tanto, este tipo de análisis es una fotografía en un momento y desde un ángulo determinado que sirve como aproximación pero que para una visión integral-holística de la realidad de esa universidad en cuanto a la incorporación de la formación en COMPINFO/ALFIN es necesario hacer un proceso de recopilación de información que implique la triangulación (diferentes fuentes tanto cualitativas como cuantitativas) que permitan lograr los otros ángulos: indagar directamente a la biblioteca sobre lo que se hace; retomar las publicaciones académicas-investigativas sobre el tema respecto a la situación de cada país y de lo que ha hecho cada biblioteca; y recibir las referencias de otras universidades sobre lo que hacen otras universidades, los casos destacados.

Finalmente, teniendo en cuenta este marco metodológico y conceptual-teórico y las aclaraciones sobre los alcances del análisis (la fotografía en un momento específico y desde un determinado ángulo) se procedió a hacer el análisis concreto de la realizad que visualizan desde sus sitios web las bibliotecas universitarias de Brasil.

\section{Análisis de la información sobre formación en COMPINFO/ALFIN de los sitos Web de las bibliotecas universitarias brasileras}

Para la realización de este análisis, el primer paso metodológico realizado, como marco contextual de este trabajo investigativo, fue una revisión bibliográfica de los artículos publicados en revistas brasileras sobre esta temática de COMPINFO/ALFIN utilizando la Base de Dados Referenciais de Artigos de Periódicos em Ciência da Informação-BRAPCI ${ }^{5}$, la cual permitió detectar (a marzo de 2011) 79 trabajos relacionados.

Tras la visualización de estos trabajos, se logró tener un marco de los avances de Brasil en este campo a nivel universitario y tomar nota de fuentes de información claves para identificar los sitios Web de bibliotecas universitarias -instituciones de educación superior- brasileras.

5Disponible en: <http://www.brapci.ufpr.br/>. Consultado: 31/12/2012.

Perspectivas em Ciência da Informação, v.17, n.1, p.134-152, jan./mar. 2012 
Web

Esto permitió elegir como fuente inicial la Comissão Brasileira de Bibliotecas Universitárias-CBBU ${ }^{6}$ que registra a la fecha 606 bibliotecas universitarias de instituciones públicas y 422 de instituciones privadas/particulares. No obstante, en esta fuente no están registradas todas las bibliotecas universitarias brasileras además se presentaban múltiples duplicaciones de instituciones y bibliotecas además de múltiples links no vigentes, por lo cual para completar y depurar ese listado, se recurrió al sitio web de la Associação Brasileira dos Reitores das Universidades Estaduais e Municipais-ABRUEM (44 Instituciones de Educación Superior) ${ }^{7}$ para garantizar la presencia en el análisis de todas las bibliotecas universitarias de carácter público ${ }^{8}$.

A su vez, con el fin de adicionar otras de las bibliotecas, especialmente las universitarias privadas/particulares de más prestigio y con presencia importante en la Web, para así alcanzar una muestra representativa de la visibilidad de COMPINFO/ALFIN en los sitios web de las bibliotecas universitarias brasileras, se recurrió a una fuente internacional como el Ranking Web de Universidades del Mundo del Centro de Ciencias Humanas y Sociales del Consejo Superior de Investigaciones Científicas de España-CCHS/CSIC ${ }^{9}$, desde el cual, se retomaron y añadieron las instituciones que no estaban reportadas ni el $C B B U$ y en $A B R U E M$, considerando las universidades/instituciones de educación superior de Brasil ubicadas entre las 6.000 primeras del total del ranking mundial ${ }^{10}$.

El análisis de estas tres fuentes generó finalmente un listado de 465 bibliotecas universitarias, 224 pertenecientes a bibliotecas de universidades públicas y 241 a bibliotecas de universidades privadas/particulares.

Para la realización de este análisis del sitio Web de cada una de estas bibliotecas universitarias, se utilizó una ficha considerando algunos postulados del análisis de contenidos (White y Marsh, 2006), en la cual se registraban los datos básicos de cada biblioteca, su url, la información sobre COMPINFO/ALFIN teniendo en cuenta las 7 macrovariables a valorar, y finalmente, considerando esa información, en cuál de los 4 niveles de incorporación de Alfabetización Informacional se encontrarían (teniendo especialmente atención en la tipología de cursos que ofrecían). 6 Disponible en: <http://www.cbbu.org/index.php?option=com content\&view=article\&id=12\&ltemid=2>.
Consultado: $31 / 12 / 2012$.
7 Disponible en: <http://www.abruem.org.br/filiadas.php>. Consultado: $31 / 12 / 2012$.
8 El análisis de las bibliotecas universitarias de carácter público se completó aprovechando la información
compartida de manera directa por la profesora-investigadora Henriette Ferreira Gomes del Instituto de Ciência
da Informação da UFBA e Coordenadora do PPGCI/UFBA, con base en los listados elaborados por su grupo de
trabajo para la publicación del informe: "A mediação da informação pelas Bibliotecas universitárias: um
mapeamento sobre o uso dos dispositivos de comunicação na web" (2010), en el marco de su investigación:
"Mediação para leitura e escrita nas atividades das bibliotecas das universidades públicas brasileiras".
Disponible en: <http://www.ies.ufpb.br/ojs2/index.php/ies/ article/view/9047>. Consultado: $31 / 12 / 2012$.
9 Disponible en: $<$ http://www.webometrics.info/>. Consultado: $31 / 12 / 2012$.

10Disponible en: <http://www.webometrics.info/rank by country es.asp?country=br>. Consultado: $31 / 12 / 2012$. 


\section{Resultados}

Tras el análisis de estas 465 bibliotecas universitarias brasileras (Anexo 1: http://bit.ly/riPgFW) se obtuvieron los siguientes resultados, que se sintetizan en los siguientes gráficos:

Figura 2 - No de bibliotecas universitárias brasileras analizadas por Estado

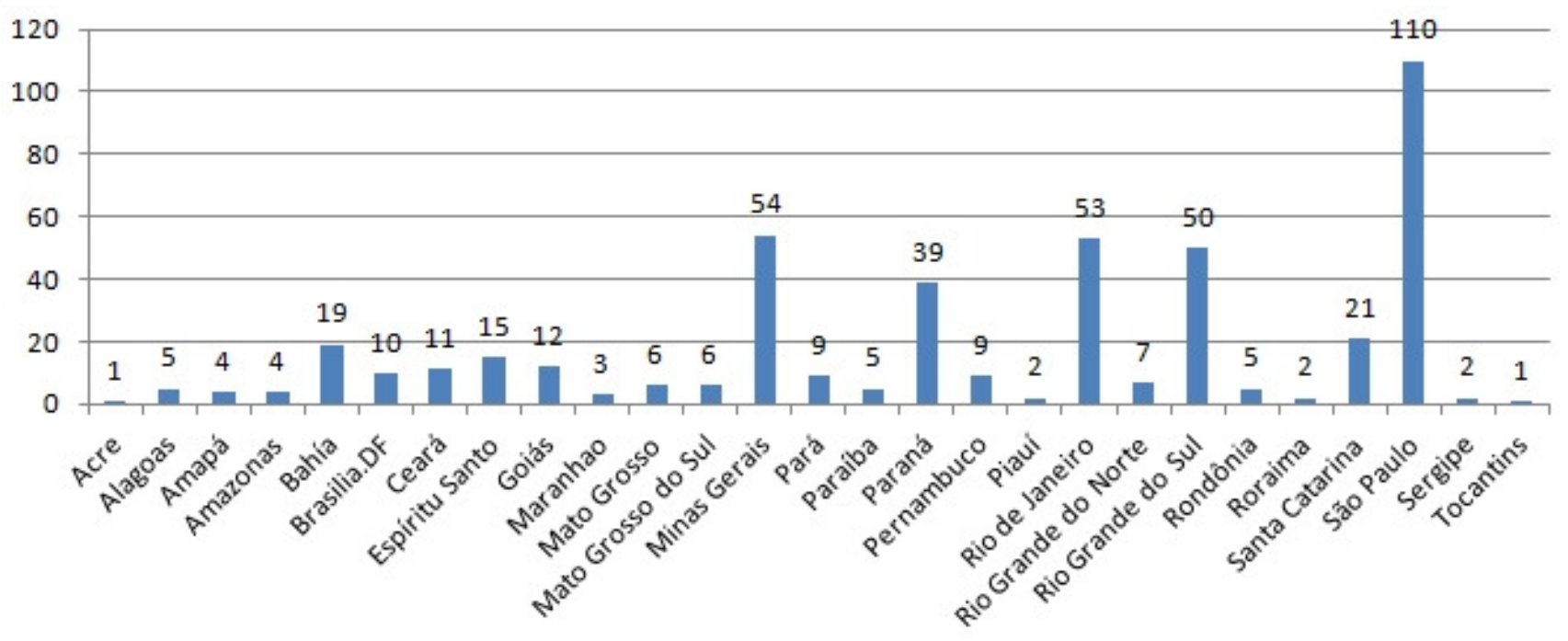

Fuente: Datos de la investigación.

Figura 3 - No. de bibliotecas universitarias brasileras presentan o no información sobre formación: F.U. o COMPINFO/ALFIN

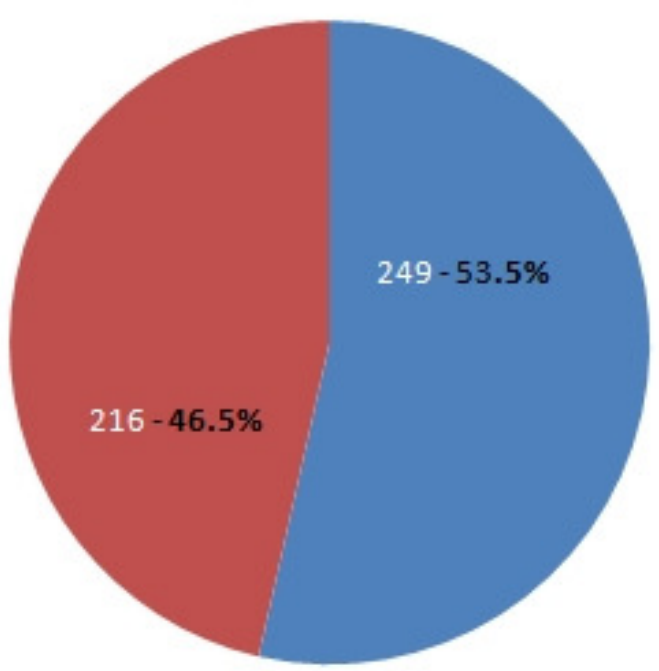

Si presentan

No presentaban

Fuente: Datos de la investigación. 
Figura 4 - Niveles de incorporación de F.U. o COMPINFO/ALFIN de las 249 bibliotecas universitarias que sí presentan información en sus sitios web sobre formación

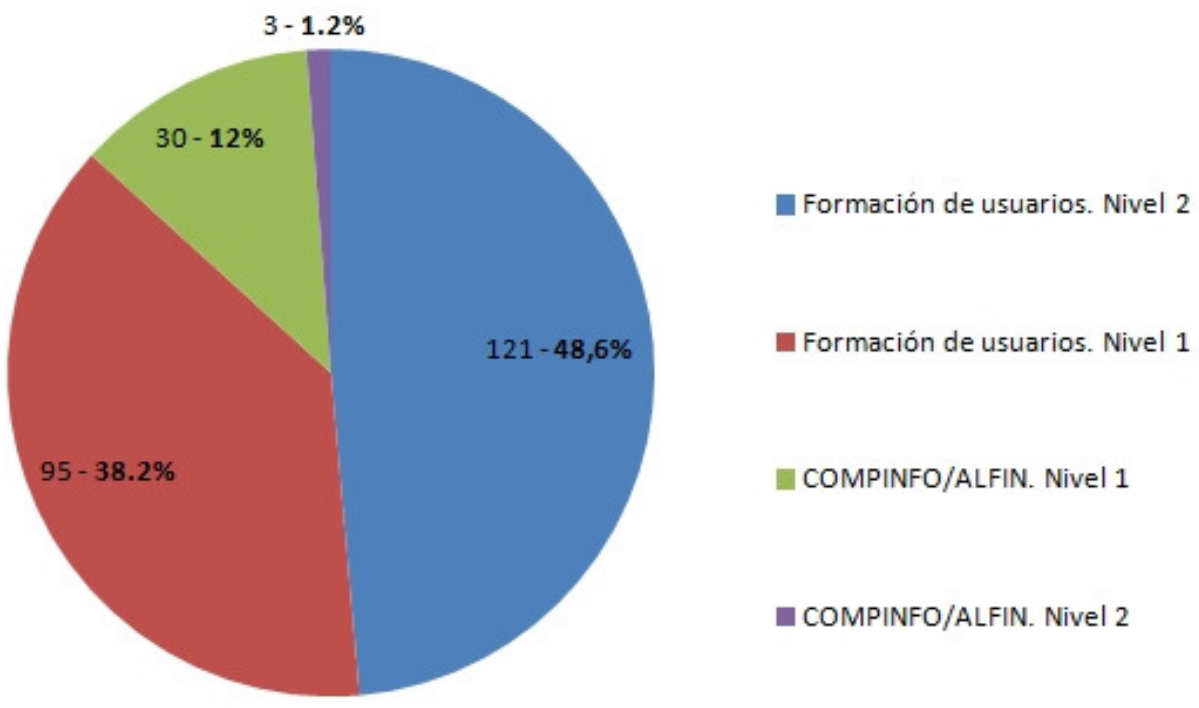

Fuente: Datos de la investigación.

Figura 5 - Tipología (pública o privada/particular) de las todas las universidades analizadas y la presencia o no de formación y los niveles de incorporación F.U o COMPINFO/ALFIN

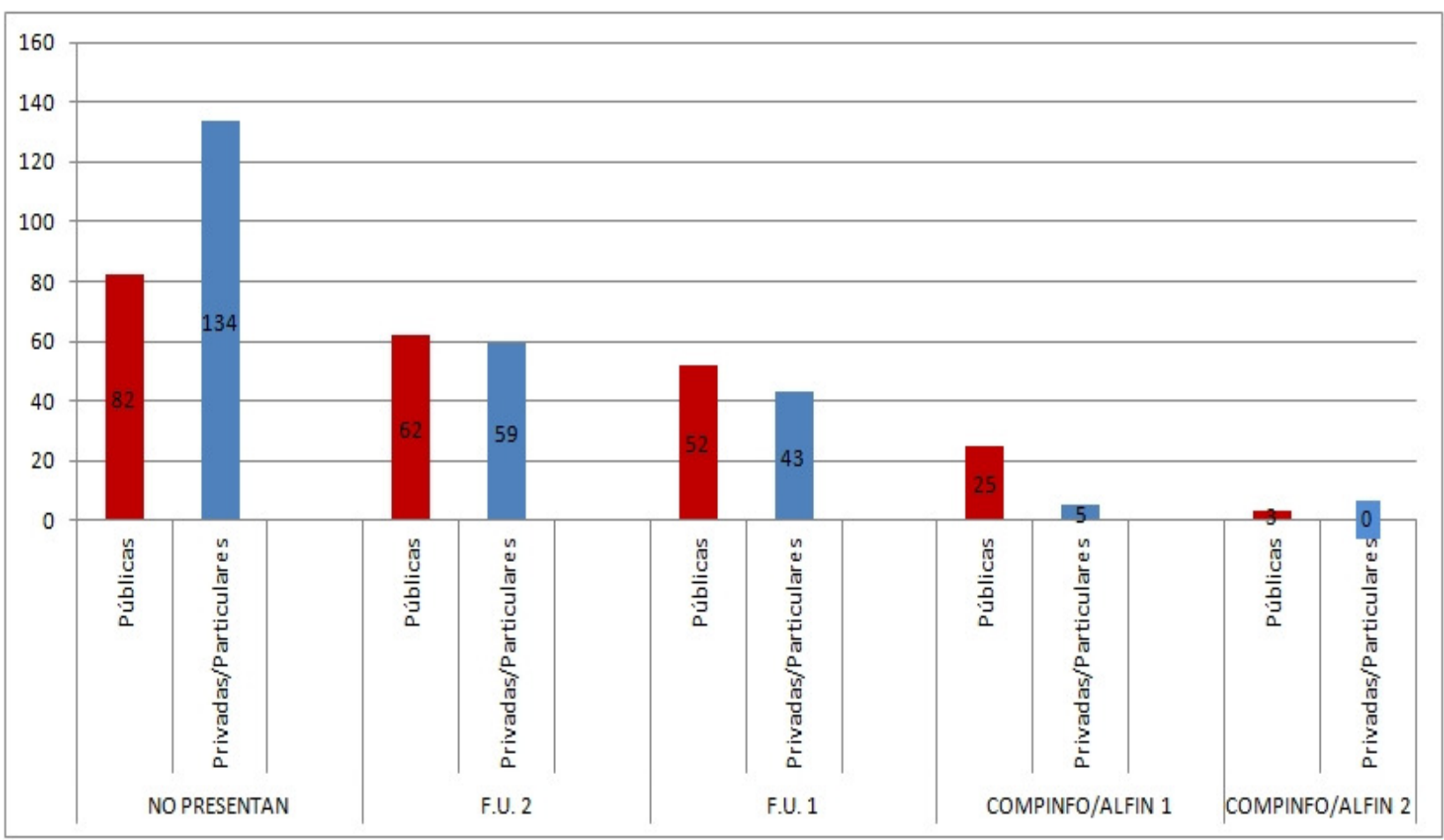

Fuente: Datos de la investigación. 
Figura 6 - Procedencia según Estados de las 33 bibliotecas universitarias que presentan niveles de incorporación de COMPINFO/ALFIN 1 ó 2

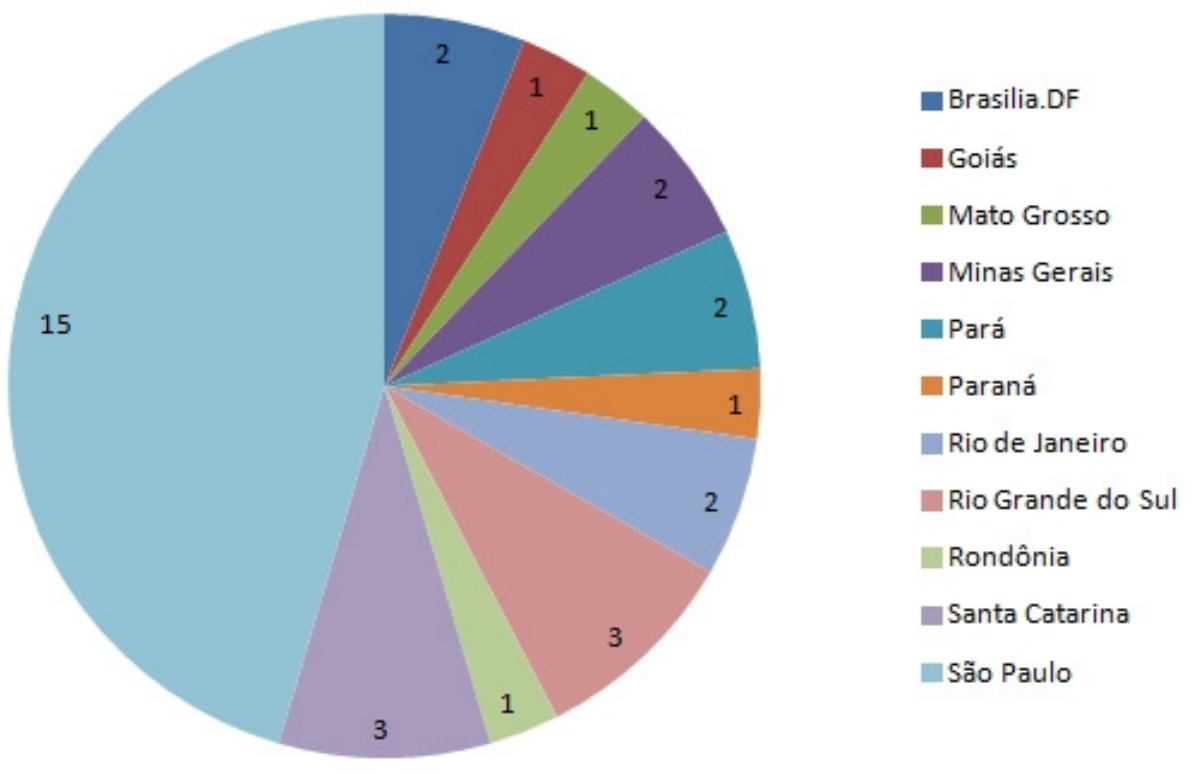

Fuente: Datos de la investigación.

Figura 7 - Comparación entre los Estados de las 33 bibliotecas universitarias que presentan niveles de incorporación de COMPINFO/ALFIN 1 ó 2

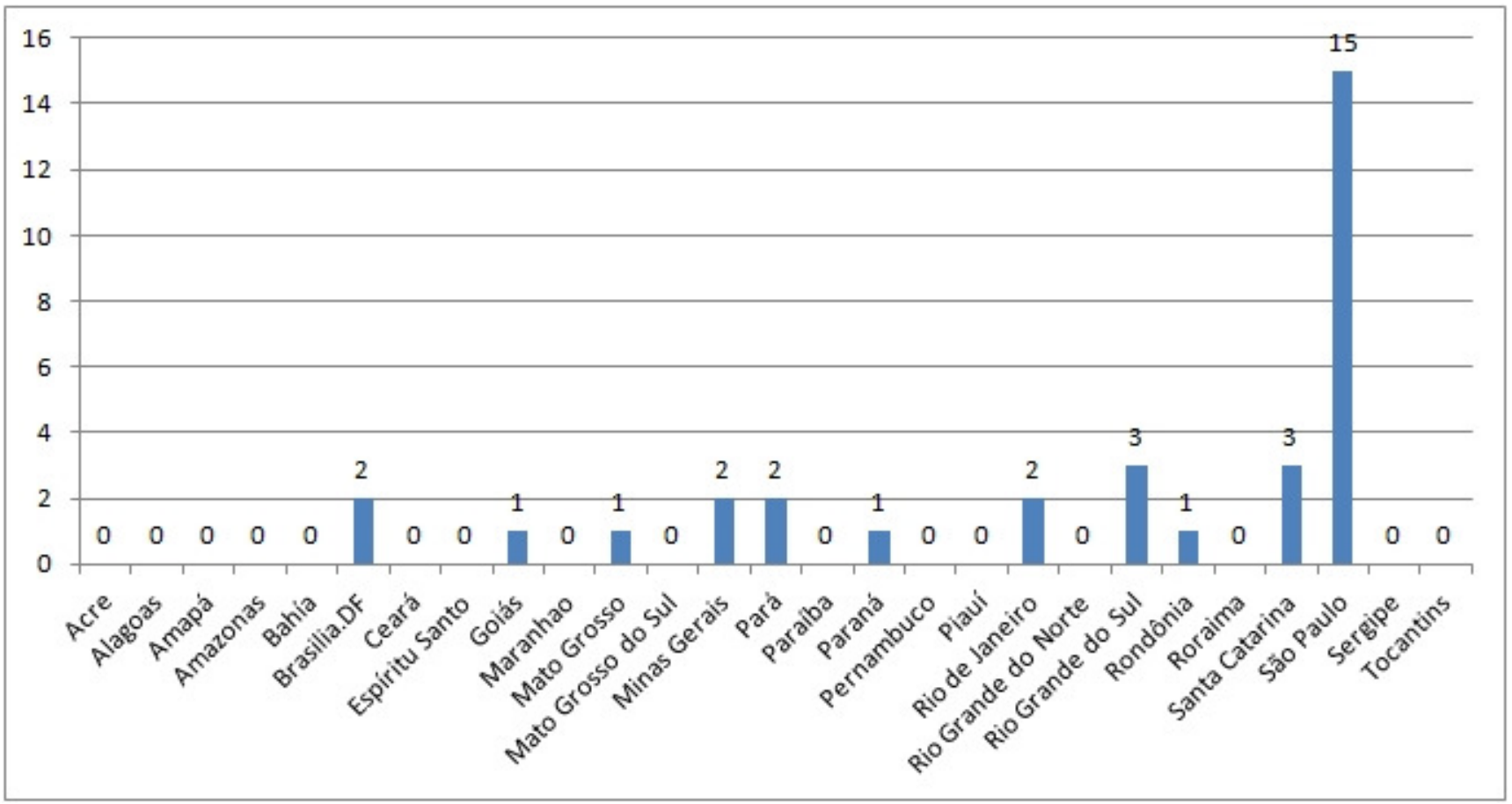

Fuente: Datos de la investigación. 


\section{Análisis general de los resultados}

Teniendo en cuenta estos resultados podemos identificar varios aspectos destacados relativos a cómo se estaría dando la incorporación de COMPINFO/ALFIN en las bibliotecas universitarias brasileras según la información de sus sitios Web:

a)hay desde la información que presentan en la Web, una concentración en los servicios más habituales (Figura 3), donde la formación no es uno de los más presentes y desarrollados: bibliotecas universitarias brasileras, el $46.5 \%$ de las analizadas, no presentan información de este tipo de servicio;

b)entre las 249 bibliotecas que sí presentan información sobre formación (figura 3), un porcentaje muy significativo se limita a actividades y cursos esporádicos centrados más en lo instrumental, es decir, el dar a conocer el catálogo y promover el uso de algunas bases de datos: F.U. nivel 2: 121 bibliotecas y F.U. nivel 1: 95 bibliotecas (Figura $4)$, que en generar formación en todas las competencias informacionales que permitan una gestión de la información y el conocimiento;

c)solamente un porcentaje muy reducido, 33 bibliotecas universitarias (figura 4) estaría presentando un nivel de incorporación 1 ó 2 en COMPINFO/ALFIN. De esas, 30 bibliotecas estarían en el nivel 1 y solo 3 bibliotecas en el nivel 2 (que implica lo curricular). De de ellas, 28 bibliotecas serían de carácter público y bibliotecas de carácter privado/particular (figura 5). Esto implica para esta última tipología de universidades (y para el Gobierno) un llamado de atención al tener un número tan reducido de bibliotecas que alcancen estos niveles 1 ó 2 en COMPINFO/ALFIN, y en general en todos los niveles analizados ya que sus resultados son los más negativos, máxime cuando se considera que porcentualmente son la mayoría de universidades existentes en Brasil;

d)a su vez, se evidencia una concentración de los casos de desarrollos de COMPINFO/ALFIN a nivel 1 ó 2 (figura 6) en un Estado, como el de Sao Paulo

(15 bibliotecas, equivalente al $47 \%$ ) y la presencia de los demás casos repartidos entre los otros 10 Estados. Esto implicaría que en total de las bibliotecas universitarias analizadas, en 16 Estados ninguna de sus bibliotecas universitarias (figura 2 y 7 ) estaría teniendo una incorporación de este tipo de nivel que implica 
una formación considerando todas las competencias informacionales que implica la Alfabetización Informacional.

\section{Consideraciones finales}

Los resultados de este análisis (de 465 sitios Web de bibliotecas universitarias brasileras) demuestran que aún hay mucho trabajo por hacer en todas las instituciones de educación superior de Brasil para que la formación en competencias informacionales, como reitera la UNESCO ${ }^{11}$, sea una de las claves para el aprendizaje para toda la vida, ya que una cantidad considerable de las bibliotecas universitarias brasileras no presentan ninguna información sobre formación o esta se limita a un nivel F.U 1 ó 2 . (216 bibliotecas, respectivamente).

No obstante, entre los 33 casos que sí presentan niveles 1 ó 2 de COMPINFO/ALFIN hay una evidencia de trabajos importantes que se vienen haciendo y que poco a poco pueden ir impactando y motivando el desarrollo en esas bibliotecas universitarias que aún no tienen programas de COMPINFO/ALFIN si se siguen políticas de trabajo colaborativo, de benchmarking, de formación interuniversitaria, de intercambio de recursos-objetos de aprendizaje, entre otras estrategias.

En ese deseo que la formación en competencias informacionales se vaya ampliando y profundizando en todas las bibliotecas universitarias brasileras, un papel fundamental lo tendrán instituciones como el Instituto Brasileiro de Informação em Ciência e Tecnologia-IBICT y sus desarrollos actuales a nivel de posgrado en diferentes programas de todo Brasil ${ }^{12}$, y la Biblioteca Nacional de Brasil en Brasilia con su proyecto de ALFIN/BRASIL ${ }^{13}$, entre otros.

A su vez, un punto clave será también, la acogida y aplicación que tenga, entre todas las instituciones educativas (primaria, segundaria, universidad), tipologías de bibliotecas y bibliotecarios (escolares, públicas, académicas, especializadas), asociaciones profesionales y escuelasfacultades de bibliotecología-ciencia de la información, la reciente "Declaração de Maceió sobre a competência em informação"14, que entre sus apartes invita a tres acciones muy concretas, que si se siguen, con seguridad en un futuro a mediano y largo plazo harán que la presencia de la formación en COMPINFO/ALFIN sea más fuerte en Brasil:

1)as escolas de formação em Biblioteconomia e Ciência da Informação deverão integrar conteúdos relativos à Competências em Informação nos seus projetos políticopedagógicos;

11Disponible en: <http://portal.unesco.org/ci/en/ev.php-

URL ID=22445\&URL DO=DO TOPIC\&URL SECTION=-465.html>. Consultado: 20/07/2009.

12 Disponible en: <http://www.ibict.br/secao.php?cat=Biblioteca\%20do\%20IBICT/fontes/capacita\%E7\%E30>. Consultado: 25/05/2011.

13Disponible en: <http://alfinbrasil.org>. Consultado: 10/08/2011.

14Disponible en:

<http://www.cfb.org.br/UserFiles/File/Declaracao\%20de\%20Maceio\%20sobre\%20Competencia\%20em $\% 20$ Informacao.pdf>. Consultado: 10/08/2011. 
2)as associações profissionais deverão dar prioridade a oferta de atividades de formação contínua, incluindo desafios decorrentes da necessidade da Competência em Informação, a fim de propiciar atualização de acordo com as tendências contemporâneas;

3)As bibliotecas, instituições, organismos e profissionais interessados no fomento e promoção da Competência em Informação deverão estabelecer relações locais, regionais, nacionais e internacionais, para a coordenação e desenvolvimento de ações conjuntas.

Finalmente, tras todo este trabajo queda otra consideración muy práctica-pragmática que deben tener muy en cuenta las bibliotecas universitarias respecto a que si esta realidad que muestran sus sitios Web, no representa todo lo que realmente se está haciendo a nivel de COMPINFO/ALFIN, debido a falta de divulgación, actualización y gestión de sus contenidos; estos resultados son un llamado hacerlo cuanto antes, por posicionamiento y visibilidad, ya que en la sociedad actual la Web es la primera puerta de imagen de una biblioteca y sus servicios: "Si no se divulga, no se conoce. Si no se conoce, no se usa. Si no se usa, tiene el peligro de desaparecer por no considerarse útil".

\section{Referencias}

DA ROCHA, C. et al. Abordagens das revistas brasileiras de ciência da informação e biblioteconomia a respeito do letramento informacional. Revista $A C B$ :

Biblioteconomia em Santa Catarina, v.
13,
$\mathrm{n}$.
1 ,
p. 145-158,
2008.
Disponible
en:

$<$ http://www.brapci.ufpr.br/documento.php?

dd0=0000008020\&dd1 $=0$ f61 bhttp://www.brapci.ufpr.br/documento.php?

dd0 $=0000008020 \& d d 1=0 f 61 b>$. Consultado: $28 / 10 / 2010$.

DUDZIAK, E. A. Competência Informacional: análise evolucionária das tendências da pesquisa e produtividade científica em âmbito mundial. Informação \& Informação, v. 15, n. 2, p. 1-22, 2010. Disponible en: $<$ http://www.uel.br/revistas/uel/index.php/informacao/article/

view/7045/6994http://www.uel.br/revistas/uel/index.php/informacao/article/ view/7045/6994>.Consultado: 18/03/2010.

FERREIRA GOMES, H.; PRUDÊNCIO, D. S.; CONCEIÇÃO, A. V. da. A mediação da informação pelas Bibliotecas universitárias: um mapeamento sobre o uso dos dispositivos de comunicação na web. Informação \& Sociedade: Estudos, v. 20, n. 3, p. 1-12, 2010. Disponible en: $<$ http://www.ies.ufpb.br/ojs2/index.php/ies/article/view/9047/4812http://www.ies.ufpb. br/ojs2/index.php/ies/article/view/9047/4812 >. Consultado: 09/01/2011.

FINQUELIEVICH, S.; PRINCE, A. Universidades y TIC en Argentina: universidades argentinas en la Sociedad de la Información. Buenos Aires: Fundación Telefónica. 2006. 
LECARDELLI, J.; PRADO, N. S. Competência informacional no Brasil: um estudo bibliográfico no período de 2001 a 2005. Revista Brasileira de Biblioteconomia e Documentação v. 2, n. 2, 2006. Disponible en: <http://www.brapci.ufpr.br/

documento.php?dd0 $=0000004329 \& d d 1=$ ce49dhttp://www.brapci.ufpr.br/ documento.php?dd0=0000004329\&dd1=ce49d > . Consultado: 28/10/2010.

MELO, A.V.; ARAÚJO, E. A. Competência informacional e gestão do conhecimento: uma relação necessária no contexto da sociedade da informação. Perspectivas em 12 , n. 2, p. 185-201, 2007. Disponible en: $<$ http://www.scielo.br/scielo.php?script=sciarttext\&pid=S1413$99362007000200012 \& \operatorname{lng}=$ es \&nrm=isohttp://www.scielo.br/scielo.php?script=sciarttext\&pid=S141399362007000200012\&lng=es \&nrm=iso >. Consultado: 09/01/2011.

SANTOS, E. M.; DUARTE, E. A.; PRATA, N. V. Cidadania e trabalho na sociedade da informação: uma abordagem baseada na competência informacional.

Perspectivas em Ciência da Informação, v. 13, n. 3, p. 208-222, 2008. Disponible en: $<$ http://www.scielo.br/scielo.php?script=sci arttext\&pid=S141399362008000300014\&lng=es \&nrm=isohttp://www.scielo.br/scielo.php?script=sci arttext\&pid=S141399362008000300014\&lng=es \&nrm=iso >. Consultado: 09/01/2011.

URIBE-TIRADO, A. Avances y perspectivas de ALFIN en Iberoamérica: una mirada desde la publicación académico-científica y la web 1.0 y 2.0 . Congreso INFO Cuba 2010-Instituto de Información Científica y Tecnológica (IDICT). p. 1-30, 2010. Disponible en: <http://eprints.rclis.org/ handle/10760/14638http://eprints.rclis.org/ handle/10760/14638>. Consultado: 2102-2011.

URIBE-TIRADO, A. La alfabetización informacional en Iberoamérica: una aproximación a su pasado, presente y futuro desde el análisis de la literatura publicada y los recursos web. IBERSID: revista de sistemas de información y documentación. Universidad de Zaragoza, p.165-176, 2010. Disponible en: <http://eprints.rclis.org/ handle/10760/14638http://eprints.rclis.org/ handle/10760/14638> Consultado: 21/02/2011.

URIBE TIRADO, A. La alfabetización informacional en la universidad: descripción y categorización según los niveles de integración de ALFIN. Caso Universidad de Antioquia. Revista Interamericana de Bibliotecología, v. 33, n. 1, p. 10-45, 2010. Disponible en:

$<\underline{\text { http://aprendeenlinea.udea.edu.co/revistas/index.php/RIB/article/view/ }}$ 
6280/5801http://aprendeenlinea.udea.edu.co/revistas/index.php/RIB/article/view/ 6280/5801>. Consultado: 21/02/2011.

URIBE-TIRADO, A. Informe-estado del arte de la alfabetización informacional en Colombia. IFLA-Information Literacy Section. 2011. Disponible en: <http://www.ifla.org/en/publications/information-literacy-state-of-theart-report-colombia-espa-olhttp://www.ifla.org/en/publications/information-literacystate-of-the-art-report-colombia-espa-ol>. Consultado: 21/02/2011.

WEBBER, S.; JOHNSTON, B. Working towards the information literate university. En: Walton, G. and Pope, A. (Eds.). Information literacy: recognising the need. Staffordshire University, Stoke-on-Trent: 17 May 2006. Oxford: Chandos. p 47-58, 2006 Disponible en: $<$ http://dis.shef.ac.uk/sheila/staffs-webberjohnston.pdfhttp://dis.shef.ac.uk/sheila/staffs-webber-johnston.pdf>. Consultado: 20/10/2009.

WHITE, M. D.; MARSH, E. E. Content analysis: a flexible methodology. Library Trends, v. 55, n. 1, p. 22-45. 2006. 